Consistent data patterns across patient demographics (age, gender, urban/rural location).

Consistent data patterns across clinical settings and Health Boards.

Conclusion. There is large appetite for VC in Wales, with high potential of sustainability and long-term use beyond COVID-19. The service is now working with clinicians, patients, carers and policy makers to explore the long-term use and sustainability of video consultations in Wales

\section{Royal Australian and New Zealand College of Psychiatrists mood disorders clinical practice guidelines update}

John Allan*

Royal Australian and New Zealand College of Psychiatrists ${ }^{\star}$ Corresponding author.

doi: 10.1192/bjo.2021.469

Aims. To provide guidance for the management of mood disorders, both depressive and bipolar disorders, based on scientific evidence supplemented by expert clinical consensus.

Background. It is the EIT responsibility to monitor a patient's physical health and the effects of anti-psychotic medication for at least the first 12 months.

Method. The update has been developed in a consistent manner to the 2015 guideline. The composition of the working group has remained largely the same as has the process to evaluate the evidence and synthesise the findings. To approach the update, the working group identified areas within the 2015 guideline where significant changes had occurred, for example the development of new therapies or where thinking and practice have changed and new ideas have emerged. Recommendations were reviewed in light of any new findings and evidence. As only some sections of the 2015 guideline have been updated/revised, the time taken to develop the update has been considerably shorter. Public consultation and peer review informed the final version.

Result. This led us to review the mechanism in the team for arranging and reviewing these investigations.

Conclusion. The mood disorders clinical practice guideline update addresses both depressive and bipolar disorders. It provides up-to-date recommendations and guidance within an evidencebased framework supplemented by expert clinical consensus.

\section{A quality improvement project (QIP) to address} communication and safety concerns from the on-call team at the Bethlem Royal psychiatric hospital out-of-hours through the introduction of weekend safety huddles

Helen Allis*, Mariam Zahedi, Thomas Stephenson, Michael Newson and Anil Kumar

South London and Maudsley NHS Foundation Trust ${ }^{*}$ Corresponding author.

doi: 10.1192/bjo.2021.470

Aims. There have been long-standing concerns about communication and safety on the Bethlem site out-of-hours due to its size, acuity and the number of specialist services; these issues were exacerbated by the COVID-19 pandemic. A Quality Improvement Project was designed to address communication and safety concerns from the on-call team at the Bethlem Royal psychiatric hospital out-of-hours through the introduction of weekend safety huddles.
Method. Daily weekend safety huddles were introduced to improve communication regarding workload, acuity, new admissions, seclusion reviews, deteriorating patients; and to improve team cohesiveness and trainee support out-of-hours.

The QIP team involved the deputy medical director, the associate director for speciality units, consultants, the college tutor, specialty registrars and core psychiatry trainees. Prior to initiating the huddles, the QIP team met to decide which specialties to involve, to agree on an agenda and liaise with other sites regarding existing huddles. Once the huddles began in April 2020, the team met periodically to agree next courses of action and to troubleshoot. The huddles initially involved acute services and eventually included CAMHS, Forensic, Older Adults, Specialist Units, all on-call consultants, the on-call registrar, two core trainees, the psychiatric liaison manager and the duty senior nurse.

Result. Data were gathered throughout the QIP using Likert scale surveys which were sent to all junior doctors on the out-of-hours rota. Paper surveys were used initially but were later replaced with Microsoft Forms to ensure anonymity.

The percentage of respondents who answered "most of the time" or "all of the time" increased across all parameters when comparing data from before and after implementation of the safety huddles.

These results included improvement in: understanding of workload and acuity ( $9 \%$ before vs $69 \%$ after), discussion of new admissions on site ( $4 \%$ before vs $90 \%$ after), discussion of patients with deteriorating mental health (35\% before vs $90 \%$ after) and physical health (22\% before vs $83 \%$ after), understanding of number of patients in seclusion (61\% before vs $93 \%$ after) and feeling part of a cohesive "on-call" team ( $17 \%$ before vs $86 \%$ after). In addition, the results suggested a reduction in frequency of safety concerns on site ( $83 \%$ answered at least "sometimes" before vs $62 \%$ after).

Conclusion. The results of the final survey demonstrated a measurable and positive impact on communications between the out-of-hours team, improved team cohesiveness and a reduction in safety concerns. The lessons learnt also influenced decisions made in formatting safety huddles at other trust sites.

\section{A quality improvement project to increase patient} feedback in the psychotherapy department,

\section{Tavistock Clinic}

Avgoustina Almyroudi ${ }^{\star}$, Alan Baban and Sukhjit Sidhu

Tavistock and Portman NHS Trust

${ }^{*}$ Corresponding author.

doi: 10.1192/bjo.2021.471

Aims. A rigorous and systematic patient feedback system is important for identifying gaps, improving the quality of care and encouraging patient involvement in service delivery. In the Adult Complex Needs Service of the Tavistock Clinic, a tertiary psychotherapy centre, only $5 \%$ of patients have provided feedback when requested. This Quality Improvement (QI) project aimed at improving the return rates of the Experience of Service Questionnaire (ESQ) and the CORE Outcome Measure by $10 \%$ within a year.

Method. The QI methodology was used to help identify factors contributing to the low response rate, including views amongst stuff about how such feedback, and the method of its delivery, might affect a psychoanalytically-informed treatment. Previously these forms were posted or handed out in person. In the first Plan-Do-Study-Act (PDSA) cycle, the method of distribution was changed by sending out the questionnaires to patients electronically, using an online survey platform. In the second PDSA cycle, the 
CORE-34 questionnaire was replaced with a shorter version, the CORE-10. This was in order to test our hypothesis that a shorter questionnaire would result in an increase in the response rates.

Result. In the first cycle of change, 197 patients were emailed for both the CORE-34 and ESQ and a total return rate of $31 \%$ was achieved. This signified an increase of $26 \%$ in the response rate. Overall more ESQ forms were completed (35\% uptake) compared to CORE-34 forms (28\% uptake). In the second cycle 199 patients were emailed with the CORE-10 and ESQ forms. The response rate was 21\% and $18 \%$ respectively. Although the response rates decreased slightly in the second PDSA cycle the results indicated that this method of distribution was capturing a greater range of patients who had not previously provided the service with this sort of feedback.

Conclusion. Sending out the outcome measures electronically and adopting shorter versions of the CORE questionnaire increased the feedback response rate significantly, and provided the service with useful data as to patients' experience of their treatment journey here.

\section{Inspiring our future psychiatrists: a quality} improvement project to optimise the medical student experience in community CAMHS settings

Brindha Anandakumar*, Lois Nunn, Fanchea Daly and Ashleigh Dale

South London and Maudsley NHS Foundation Trust ${ }^{*}$ Corresponding author.

doi: 10.1192/bjo.2021.472

Aims. To improve the structure, quality and experience of medical student placements in Community Child and Adolescent Mental Health Services (CAMHS). To increase the likelihood of pursuing a career in Psychiatry or CAMHS by $50 \%$ over their 3 week placement.

Background. There is evidence in the literature of the widely variable medical student experiences when it comes to Psychiatry placements. Medical students from Kings' College London (KCL) have a 3 week placement in Lambeth Community CAMHS services. Despite this being a good opportunity for learning and development, the feedback from students reports that they often feel lost and were unable to fulfil the potential of the placement. The main challenges reported were identifying beneficial educational experiences and gaining clinically relevant exposure. This exposure includes getting involved beyond observation and following a patient longitudinally. These challenges will likely have a knock-on effect on their attitude towards Psychiatry and overall enjoyment of CAMHS placements when there is already a struggle to recruit trainee Psychiatrists.

Method. A structured and immersive educational placement was designed through consultation with previous students, the multidisciplinary team and the university program directors. This included:

- A new induction

- Having a role in initial assessments of young people

- Formalised medical and psychology teaching

- Communication sessions

- Case discussions in a 'grand round' format providing opportunity for end of placement assessment

Feedback was gathered using the Qualtrics analytical software, which was easily accessible through student's mobile devices.

Pre placement questionnaires were used to assess the student's initial level of knowledge, expectations from the placement and motivation or interest in a career in CAMHS. Post placement questionnaires were used to assess any change in the above baseline scores. Brief, online feedback was collected after every clinical activity and was used to assess the interest and utility of each attended session during the placement. The questionnaire feedback was analysed and using these data, adjustments were made to improve the program for the next students in a "Plan-Do Study-Act" quality improvement methodology format. We analysed whether improving placement experience and learning affected students' interest in careers in Psychiatry.

Result. The Quality Improvement Project is currently on-going and results are pending. So far, there is an improvement in student attendance and engagement following the introduction of induction, structure and active involvement in case management. Conclusion. The COVID-19 pandemic has resulted in community services having significantly reduced face to face contact, therefore our proposed changes for future cycles include various virtual elements. Ensuring medical students have access to online platforms such as Microsoft teams is vital in ensuring an effective medical student placement can be established Although the change to more remote working has been challenging, it is vital that medical students gain appropriate clinical experience during their Psychiatry placement to support further developments in Psychiatric recruitment.

\section{Standards for lithium monitoring. "Are we good at adhering to these standards in Lanarkshire"?}

Saba Ansari*, Khalid Nawab, Niall Killeen, Elizabeth Spence and Monika Fotedar

NHS Lanarkshire

${ }^{*}$ Corresponding author.

doi: 10.1192/bjo.2021.473

Aims. The National Institute for Health and Clinical Excellence (NICE) recommend that renal and thyroid function must be checked before lithium is prescribed and for all patients who are prescribed lithium should have their renal and thyroid function checked every six months, and their serum lithium checked every three months. The aim of this audit is to ascertain whether or not routine blood monitoring for bipolar disorder patients, taking lithium is in keeping with the guidelines.

Background. Lithium has been a mainstay in the management of bipolar disorder since the 1970's; indeed, lithium carbonate was first used in the early 1880's for the treatment of mania. Despite its usefulness however, the drawback of lithium treatment remains its very narrow therapeutic index, toxic side effects and as such its need for close therapeutic monitoring.

Method. A list of patients with a diagnosis of bipolar disorder being treated with lithium was collated from an electronic database of psychiatry patients in Cumbernauld Community and inpatients at Glencairn unit Coathill Hospital and Cleland Hospital. A retrospective analysis using Clinical Portal was conducted looking at blood results; Lithium levels checked 3 times a year and Kidney functions and Thyroid function checked twice a year, over the previous year. Our results were then compared to the NICE Guidelines for lithium monitoring to see if they complied with the expected routine monitoring schedule. We may have missed patients open to Community Psychiatric Nurses (CPN) but not open to Consultant psychiatrists. Other group that might have been missed could be open to General Practitioners but not to secondary care. We attempted to contact them but this was unsuccessful. 